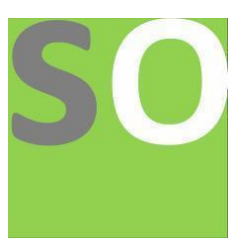

Article title: To Analyse the Resistance and Tolerance of several Multi Drug Resistant (MDR) Bacteria: How they manage to escape the Antibiotics?

Authors: Amartya Nandi[1], NAVNEET BATRA[2], KANAV SHARMA[3], BHASKAR R. PURI[4], BHAVANA M. PURI[5], KASH Affiliations: Department of Microbiology, G.G.D.S.D, PANJAB UNIVERSITY, CHANDIGARH, INDIA[1] Orcid ids: 0000-0001-6024-1699[1]

Contact e-mail: amartyanandi.research20@gmail.com

License information: This work has been published open access under Creative Commons Attribution License http://creativecommons.org/licenses/by/4.0/, which permits unrestricted use, distribution, and reproduction in any medium, provided the original work is properly cited. Conditions, terms of use and publishing policy can be found at https://www.scienceopen.com/.

Preprint statement: This article is a preprint and has not been peer-reviewed, under consideration and submitted to ScienceOpen Preprints for open peer review.

Funder: DEPARTMENT OF BIOTECHNOLOGY (DBT), GOVT. OF INDIA

DOI: 10.14293/S2199-1006.1.SOR-.PPHRVNB.v1

Preprint first posted online: 19 September 2021

Keywords: antimicrobial resistance, multidrug resistant, antibiotics, beta lactams, macrolides, biofilm tolerance, bacterial resistance, MDR, AMR, bacteria 


\title{
To Analyse the Resistance and Tolerance of several Multi Drug Resistant (MDR) Bacteria: How they manage to escape the Antibiotics?
}

Amartya Nandi ${ }^{*}$, Kanav Sharma ${ }^{2}$,Kashish ${ }^{3}$, Bhaskar R. Puri, Bhavana M. Puri, Dr. Navneet Batra',

1, 2, 3, 4, 5 Department of Microbiology \& Biotechnology, G.G.D.S.D, Panjab University, Chandigarh, India.

${ }^{6}$ Head of the Department of Microbiology \& Biotechnology, G.G.D.S.D. Panjab University, Chandigarh, India.

*Corresponding Author

\begin{abstract}
Antimicrobial resistance has become a serious problem for physicians around the world when it comes to treating patients. As a result, short-term research was carried out by us to determine the prevalence of multidrug-resistant (MDR) bacterial isolates and to study the mechanism of escaping the antibiotics. Methods and Materials In the department of microbiology, clinical samples were grown and bacterial strains were discovered. To determine we have studied several strains of clinically established MDR bacteria and analyzed their genome sequences to get the answers to the tolerance and resistance of MDR bacteria.

Keywords: antimicrobial resistance, multidrug resistant, bacterial resistance, MDR, AMR, bacteria, antibiotics, beta lactams, macrolides, biofilm tolerance.
\end{abstract}

\section{INTRODUCTION}

Researches over last three decades have reported a large number of resistances towards the antimicrobials with the evolution of normal species to multi-drug resistance (MDR) strains. This antimicrobial resistance is accompanied and encoded by genomic changes ranging from point mutations to horizontal import of genes from the environment. This is also a point of concern by WHO and several other European and American institutions. Consequently, increasing antimicrobial resistance and a decrease in new drug development due to the lack of research may result in a perfect storm for the next pandemic. This can be quoted as the best example of the purported (POST ANTIBIOTIC ERA). Thus, it is clear that effective solutions and productive research is the need of the hour. New policies and actions are necessary to avoid more than 10 million deaths worldwide by 2050 (O'Neill, 2016). 
Antibiotic-resistant bacteria can be acquired by gene mutation and horizontal gene transfer, as well as intrinsic activity deriving from inherent structural or functional features (fig 1). This is exemplified by the unavailability of a certain antibiotic's susceptible target. Gram-negative bacteria's cytoplasmic membrane seems to have a smaller proportion of anionic phospholipids than Gram-positive bacteria's. The Ca2+-mediated insertion of daptomycin into the plasma membrane, which would be crucial for its antibacterial action, is reduced as a result (Randall et al. 2012). Some Gram-negative bacteria possess protective mechanisms encoded within their DNA that allow them to tolerate antibiotic effects such as antibiotic transfer over the outer membrane. The glycopeptide antibiotic vancomycin, For example, inhibits peptidoglycan crosslinking by binding to target D-Ala-D-Ala peptides; this effect is enhanced in Gram-positive bacteria. Whereas, Drug transport across the outer membrane is impeded in Gram-negative organisms, and access to peptides in the periplasm is restricted.

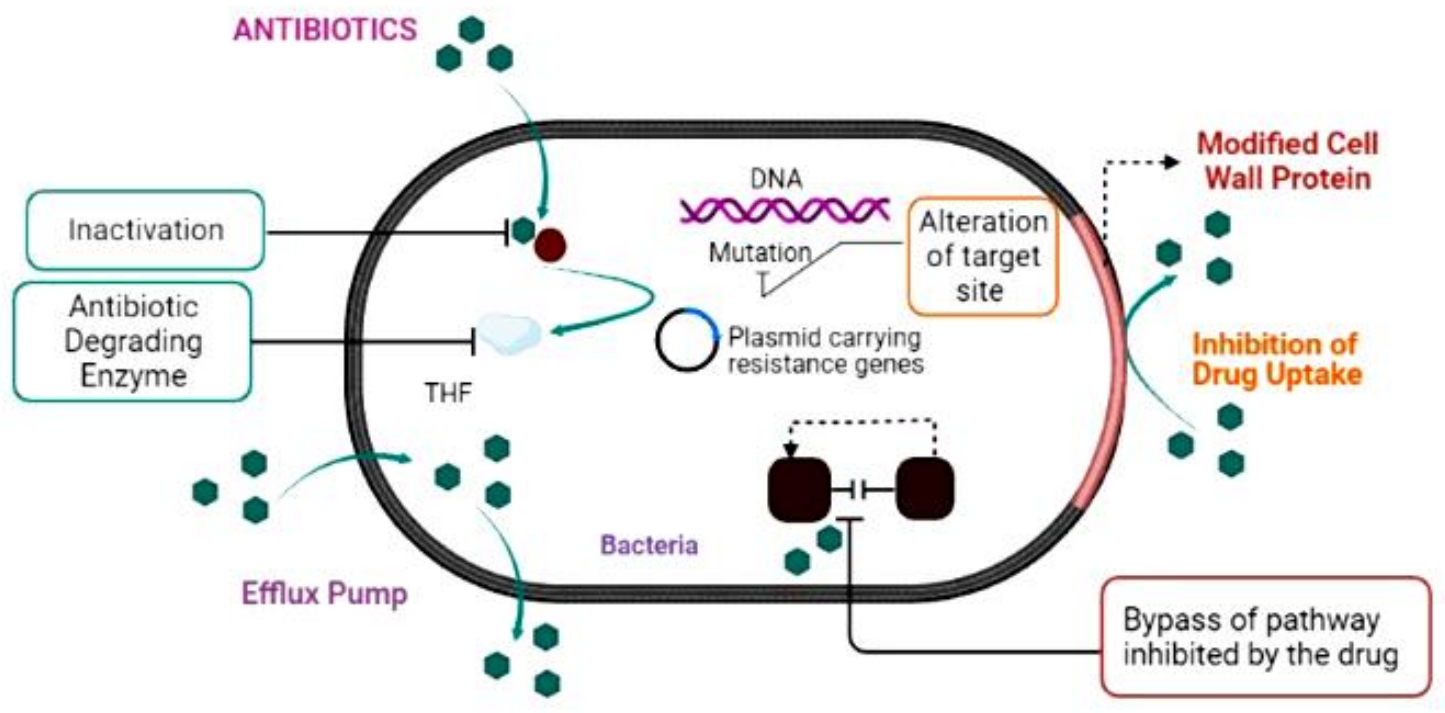

Fig 1: Evolution of Bacterial Cell to MDR Bacteria.

Antimicrobial resistance (AMR) has increased both mortality as well as morbidity from treatment failures and increase in healthcare costs. However, overuse of antibiotics exerts a driving force in the evolution of antimicrobial resistance genes causing the emergence of a huge number of multi-drug resistance strains throughout the world. The relation between resistance and increased rate of antimicrobial use has been documented for nosocomial infections and resistant community-acquired infections. As resistance develops to first-line antibiotics the process of resistance continues to a broader spectrum with reinforcement of higher grades of antibiotics followed by the development of resistance to the whole class of drugs (Feldgarden et al. 2019). 
Resistance factors can spread rapidly within the human-animal barrier. MDR strains can travel globally with newly introduced pathogens spreading rapidly in susceptible hosts (TARASI et al. 1997). The pattern of antibiotic resistance may vary from place to place, so surveillance data needs to be collected and analyzed from sentinel sources. This can help to formulate, monitor, and identify the prevailing and emerging problems which can be a possible indicator of appropriate or inappropriate antibiotic usage by clinicians in that area. Further, that can contain by effective strategy (Asia 2011).

The results of in-vitro antibiotic susceptibility testing followed by genomic identification and characterization of several multi-drug resistance bacteria using the sequence-based approach to detect AMR requires bio-informatics tools to analyze and visualization the genomic structure of the microbial resistome (CARTWRIGHT et al. 2013). Such as sequence and structural alignment, to generate new predictions or find novel biology from enormous numbers of biological data, such as genetic sequences, cell populations, or protein samples. Furthermore, the amount of data generated in the form of databases and literature for constructing molecular profiles and collecting data on drug response has grown tremendously (Carriço et al. 2013). As a result, bioinformatics tools and techniques are necessary to counter bacterial resistance, pathogen identification and type, identifying biomarkers for early diagnosis and treatment, enabling personalized interventions, and anticipating clinical outcomes (Pokrovskaya and Baasov 2010). We review previous studies, molecular and structural insight of bacterial resistance to antibiotics, and the current bioinformatics approach throughout this paper.

\section{METARIALS AND METHOD}

2.1. Isolation and Collection of Microbial Samples: Microbial samples (strains) of multi drug resistant has been ordered from the Microbial Type Culture Collection and Gene Bank (MTCC), Institute of Microbial Technology (IMTECH), Department of Biotechnology (DBT) and the Council of Scientific and Industrial Research (CSIR), Government of India facility. Then the freeze - dried samples has been rejuvenated in specified nutrient medium, incubated at $37^{\circ} \mathrm{C}$ for $24-48$ hours to perform AST test. (Table 1) contains the information of the microbial samples.

\begin{tabular}{|l|l|l|l|l|}
\hline & Name of the Strain & $\begin{array}{l}\text { MTCC No./ATCC } \\
\text { No./ NCIB }\end{array}$ & Isolated From & Special Features \\
\hline 1 & Escherichia coli & $\begin{array}{l}\text { MTCC }-443, \\
\text { ATCC }-25922, \\
\text { NCIB }-12210\end{array}$ & $\begin{array}{l}\text { Clinical } \\
\text { pathogenic } \\
\text { isolate from } \\
\text { ATCC }\end{array}$ & $\begin{array}{l}\text { Control org. for sensitivity } \\
\text { testing of bacteria to sodium } \\
\text { cephalothin, cephaloridine, } \\
\text { standardized assay including } \\
\text { antibiotic disc susceptibility } \\
\text { testing and MIC. }\end{array}$ \\
\hline 2 & $\begin{array}{l}\text { Pseudomonas } \\
\text { aeruginosa }\end{array}$ & $\begin{array}{l}\text { MTCC }-424, \\
\text { ATCC }-25619\end{array}$ & $\begin{array}{l}\text { Clinical } \\
\text { pathogenic } \\
\text { isolate from } \\
\text { ATCC }\end{array}$ & $\begin{array}{l}\text { Assay of carbenicillin } \\
\text { Assay of vancomycin } \\
\text { Opportunistic pathogen } \\
\text { research, (Pharmaceutical) }\end{array}$ \\
\hline
\end{tabular}




\begin{tabular}{|c|c|c|c|c|}
\hline 3 & $\begin{array}{l}\text { Staphylococcus } \\
\text { aureus }\end{array}$ & MTCC - 9760 & $\begin{array}{l}\text { Metagenomic } \\
\text { Sample from } \\
\text { Salt mines of } \\
\text { Panchpada, } \\
\text { Barmer, } \\
\text { Rajasthan, } \\
\text { India. }\end{array}$ & N/A \\
\hline 4 & Bacillus subtilis & MTCC -1133 & $\begin{array}{l}\text { Clinical } \\
\text { sample from } \\
\text { P. Sharma, } \\
\text { Dept. of } \\
\text { Microbiology, } \\
\text { Panjab } \\
\text { University, } \\
\text { Chandigarh, } \\
\text { India }\end{array}$ & Genetic stock, thr5, trpC2 \\
\hline
\end{tabular}

2.2. Antibiotic Susceptibility Testing (Kirby and Bauer Test): The determinations of efficacy of a noxious agent to bacteria were well denoted by Walker, Rideal and others. Alexander Fleming used the ditch plate method which was devised by some field workers.

With the introduction and development of antibiotics, it is necessary to perform AST at routine for updating information as when it's required. Where, antibiotics contained in a reservoir are allowed to diffuse into the medium to interact with recently incubated microbial culture. In this case absorbent paper discs play the role of reservoir, most prominent and easy method to perform AST.

\subsubsection{Reagents for Kirby and Bauer Test:}

Mueller - Hinton Agar (Himedia): $38 \mathrm{~g}$ in 1000mL of distilled water.

Beef extract $-2.00 \mathrm{~g}$, Acid hydrolysate of casein - 17.50g, Starch - 1.50g, Agar - 17.00g, Distilled water-1000mL; pH-7.3 $\pm 0.1 @ 25^{\circ} \mathrm{C}$.

0.5 McFarland Solution: $0.5 \mathrm{~mL}$ of $0.048 \mathrm{~mol} / \mathrm{L} \mathrm{BaCl} 2\left(1.175 \% \mathrm{w} / \mathrm{v} \mathrm{BaCl} 2.2 \mathrm{H}_{2} \mathrm{O}\right)$ is added to $99.5 \mathrm{~mL}$ of $0.18 \mathrm{~mol} / \mathrm{L} \mathrm{H}_{2} \mathrm{SO}_{4}(1 \% \mathrm{v} / \mathrm{v})$; the absorbance at $625 \mathrm{~nm}$ should be between $0.008-0.10$.

Antibiotics (discs): Readymade cartridges containing antibiotic discs have been ordered from Himedia Pvt. Ltd. Discs should be stored at $4^{\circ} \mathrm{C}$ or below, some antibiotics like $\beta$-lactams, cephalosporins and cluvanic acid are recommended to store at $-14^{\circ} \mathrm{C}$ in a non frost freezer until needed.

\subsubsection{Procedure for performing Kirby and Bauer Test:}


Inoculum preparation: The freeze dried culture which has been ordered were revived in their specific growth medium and incubated for $35^{\circ} \mathrm{C}$ for $24-48$ hours. The incubated broth is then compared with the turbidity of $0.5 \mathrm{McF}$ arland standard with a use of spectrophotometer, if found more the turbidity of broth should be adjusted by adding sterile saline in the broth.

(Approximately 1 to $2 \times 10^{6} \mathrm{CFU} / \mathrm{mL}$ ).

Inoculation of test plates: Ideally, within 15 minutes of turbidity adjustment of inoculum suspension, it is recommended to inoculate the Mueller Hinton plates with a help of sterile cotton swab. The swab should be rotated several times and pressed firmly on the inside walls of above the fluid level to remove the excess inoculum. The lid should be left ajar for 2-3 minutes, so that excess moisture can be absorbed.

Application of discs to inoculated agar plates: The readymade antibiotic discs should be dispensed on the surface of the inoculated Mueller Hinton agar plates and must be placed evenly so the zones are not overlapping each other. Then the plates are incubated inverted at $35^{\circ} \mathrm{C}$ for 12-24 hours.

\subsubsection{Observation of plates and interpretation of results:}

After the incubation each plate has been examined and if plate found to be satisfactorily streaked and inoculum was correct then resulting zones of inhibition should be in a uniform pattern with a confluent lawn of growth. Then the inhibition zones can be measured by the zone scale to interpret accurate results.

Results of AST are as follows: (Table 2).

2.3. Genomic Analysis Using Bio-informatics Tools: The whole genome sequence of the specified bacterial strains has been obtained from ATCC and MTCC for their further genomic analysis. Whole genome sequences of each organism have used to perform BLAST search at NCBI and then using NCBI, Pubmed and Uniprot database, we identified the specific genomes responsible for the resistance and tolerance against specific antibiotics. (Table 3) contains the list of specific genomes responsible for the resistance against specific antibiotics.

\section{DISCUSSION}

\subsection{MECHANISM OF TOLERANCE}

The mechanisms of antibiotic tolerance explained here are based on the work with P.aeroginosa, however, it is also relevant for biofilms formed by other microorganisms such as E.coli, S.aureus, B.subtilis,etc.. (Hall and Mah 2017). 


\subsubsection{Physiological Tolerance}

Physiological tolerance of bacteria is denoted by slow growth which remains for a long period of time also called as persisters. Persisters can be defined as small subpopulations of extremely tolerant, presumably dormant cells characterized by a viable distribution of metabolic activity. Which found to be remain viable after a susceptible number of populations is killed by bactericidal antibiotic. As such they have been classified as hetero tolerance and this heterogeneity is due to consumption of nutrients and oxygen by the metabolically active bacteria located at the edges of the bio-film leaving minimum or no oxygen and nutrients for the bacteria present in the inner region of the bio-film (Stewart et al. 2016).

The impact of low metabolism is the inactivation of major inactivation of antibiotic targets, thus affecting degrees of efficacy of all bactericidal antibiotics. For e.g. The effectiveness of the inhibitors of protein synthesis is altered by low protein synthesis, whereas low DNA synthesis affects the impact of quinolones and the impact of beta lactams is affected by the low peptidoglycan production.

In contrast to target inactivity, some antibiotics have a poor effect on bacteria with reduced metabolic activity, which would be induced by adaptive response to stress such as SOS and Strignet responses (Bernier et al. 2013, Nyuyen et al. 2011). The Strignet response is an evolutionary adaptation to nutrition and iron deficiency which has been demonstrated to play a key role in tolerance (Nyuyen et al. 2011). Furthermore, it has been shown that activating the Strignet response prevents the buildup of HAQ pro-oxidants (Schafhauser et al. 2014). The SOS response, on the other hand, might be a crucial mechanism for bacterial survival under stressful situations. Transcriptional repressor LexA and DNA binding activating protein RecA are found to be involved in the SOS response (Bernier et al. 2013).

Some other adaptive starvation response to food constraint has been discovered, including the catabolite repressor control protein crc, which reduces the metabolic activity of bacterial species. In comparison to wild type biofilms, crc mutant biofilms were more susceptible to ciprofloxacin and more tolerant to colistin (Zhang et al. 2012). As a result, the metabolically inactive subpopulation in biofilms doesn't really develop entirely as a direct consequence of nutritional circumstances and subsequently differentiate into metabolically inactive subpopulations. Another reason for the decline oxygen tolerance is that oxygen molecules are required for the production of ROS (hydroacyl radicals), which have been demonstrated to be crucial for antibiotics' bactericidal action (Dwyer et al. 2015).

Tolerance alterations might be triggered by antibiotic therapy or develop naturally. In any case, the tiny number of tolerant mutants would remain hidden and undiscovered in the colony under normal growth circumstances, but would survive better when the antibiotic is present, allowing them to take over the colony during antimicrobial therapy. After numerous cycles, the population would obtain greater and higher survival rate against the antibiotic, which can be directly 
attributed to the invasion of the tolerant mutant's minor population. Because the mutant has an increased proclivity to convert to persister cells, the surviving survivors would have a higher proportion of the mutant following extended treatments. When the mutant subpopulation is allowed to regenerate, it will make up a greater proportion of the cells than in the previous cycle.

\subsubsection{Physical Tolerance}

Antibiotics are always used in the process to cure infections, where they have been subjected to penetrate the outer layer (extra-cellular matrix) of the bio-film in order to reach the bacteria. Generally, bio-film matrices do not inhibit the diffusion of antibiotics. But, in some cases antibiotics are restricted to penetrate the extracellular matrix, where the antibiotic binds with the protein molecules of the bacterial membranes (Walters et al. 2013).

Antimicrobial tolerance caused by restricted penetration may be only temporary. Whereas, it will provide enough time for bacteria to adapt to a more tolerant state. Further, the mechanisms may be associated with the infections, where at the site of infection, the concentrations of antibiotics are low. So, that the bio-film matrix cannost be saturated by the antibiotics.

(Staudinger et al. 2013) stated in his report that P.aeruginosa were independent of the production of exo-polysaccharides in viscous environments that restrain motility, then wild type and an exo-polysaccharides deficient mutant strain displayed the same level of antibiotic tolerance. Based on these results we can comply that extracellular matrix does not play a role in antibiotic tolerance. However, it was also demonstrated that the biofilm matrix over expression can significantly protect against antimicrobial treatment (Goltermann and Tolker Nielsen 2017).

\subsection{RESISTANCE AgAinst PARTICULAR GROUP OF ANTIBIOTICS BY THE EXPRESSION OF SPECIFIC GENOMES}

\subsubsection{Specific Genes to Beta Lactams}

$\beta$ lactams represent one of the most major groups of modern day antibiotics which stops bacterial growth by inhibiting transpeptidase that are indispensable through the cross linking process of cell wall biosynthesis. $\beta$-lactams constitutes three carbon and one nitrogen ring which is highly reactive in nature. Due to the presence of one nitrogen ring it is also known as betalactam ring. This group of antibiotics includes penicillins, cephalosporins, carbapenems, monobactams and $\beta$ lactamase inhibitors. The mode of action of $\beta$-lactams can be grouped in 5 categories: Inhibition of cell wall synthesis; Impairment of cytoplasmic membrane; Inhibition of nucleic acid synthesis; Inhibition of protein synthesis; metabolic antagonist action. Generally, we have four basic mechanism which provides resistance to drugs in bacteria: Alteration of antimicrobial target due to either, complete loss of affinity or simple reduction; Reduction in 
amount of antimicrobial that reaches to the target by entrance reduction caused by decrease in permeability due to porin mutation or by an increase in pumping of an efflux transporter; The presence of an enzymatic mechanism that completely or partially destroys the antimicrobial molecules and the development of an alternative metabolic pathway(Westblade, Errington, and Dörr 2020).

In general, $b$ lactams have poor antimicrobial effect. This is due to the mode of action of targeting peptidoglycan synthesis. Thus the primary tolerance mechanism of $b$ lactam is related to slow growth. Resistance to these antibiotics is mediated by production of group of enzymes referred as' $\beta$ lactamases'(Dever 1991),

Extended-spectrum cephalosporin-resistant Gram-negative bacteria typically produce AmpC type beta-lactamases. AmpC beta-lactamases can be mediated by chromosomes or plasmids. Except for cefepime and carbapenems, all $\beta$-lactam antibiotics are hydrolyzed by plasmidmediated AmpC - lactamases. AmpC genes produced from plasmids are obtained from inducible chromosomal genes that have been dispersed among numerous organisms. These plasmidmediated enzymes offer a resistance pattern comparable to chromosomal AmpC-lactamases overproduction. Extended-spectrum beta-lactamases (ESBLs) are plasmid-based enzymes that provide resistance to a wide range of beta-lactam antibiotics. ESBL are categorized into three groups: TEM, SHV or CTX-M. Because of their wider range of activity, these enzymes were termed extended-spectrum-lactamases. The mutations that change a limited number of essential amino acids expand the active site of the enzyme, allowing it to deflect the oxyimino replacements that typically shelter the beta-lactam ring. As a consequence, although the traditional TEM and SHV and CTX-M enzymes are unable to hydrolyze the antibiotics, but the mutants can, imparting resistance to their host strains (Sulaiman and Lam 2021).

Beta-lactamases of the AmpC type can also be found on plasmids. Unlike ESBLs, AmpC betalactamases hydrolyze broad and extended-spectrum cephalosporins (cephamycins and oxyiminobeta-lactams), but are not inhibited by beta-lactamase inhibitors such clavulanic acid.

AmpC enzymes are inducible and can be mutated to produce high levels of expression. Resistance to broad-spectrum cephalosporins such as cefotaxime, ceftazidime, and ceftriaxone is conferred through over expression. Many antibiotics have hydrolytically sensitive chemical bonds, such as amides and esters. Several enzymes, such as Extended-spectrum-lactamases (ESBLs), are known to sabotage antibiotic activity by cleaving these bonds. Peptidoglycan synthesis in the presence of beta-lactams is performed by mecA, a foreign PBP2a acquired by lateral gene transfer.PBP2A has a lesser affinity for beta-lactam antibiotics like methicillin and penicillin than DD-transpeptidase, and therefore it doesn't bind to the penicillin-like drugs' ringlike structure. AmpC-lactamases can be mediated by chromosomes or plasmids. Except for cefepime and carbapenems, all $\beta$-lactam antibiotics are hydrolyzed by plasmid-mediated AmpClactamases. AmpC genes produced from plasmids are obtained from inducible chromosomal genes that have been dispersed among numerous organisms. These plasmid-mediated enzymes 
offer a resistance pattern comparable to chromosomal AmpC-lactamases overproduction. ampD (cytosolic amidase) helps in overexpressing ampC by reducing concentration of anhydro-UDPMurNAc tri-, tetra- and pentapeptides preventing displacement of UDP-MurNAc pentapeptide from AmpR.

PBPs involved in cell wall production are binded and inactivated by methicillin and its equivalents. Overproduction of $\beta$-lactamases, increased amounts of intrinsic PBPs, or a loss in their binding affinity are all common causes of low-level resistance. The production of an alternative PBP (PBP2a) produced by the mecA and mecCgene, which has a poor affinity for most -lactam antibiotics, is always required for high-level resistance. An extra penicillinbinding protein is encoded by the mecA determinant i.e. PBP2a. A poor rate of acylation of $P B P 2 a$, as well as a poor affinity of the enzyme for $\beta$-lactams, are known to exhibit resistance. PBP2a has a low acylation rate when it comes into contact with -lactams, according to structural studies This is owing to a deformed active site caused by the flexibility of the nonbinding (NB) domain and areas around the active site groove in the transpeptidase (TP) domain. Furthermore, Ser403's position is critical for the $\beta$-lactam ring's nucleophilic attack, which finally led to acylation of the protein. However, bcl are class A-beta lactamases, Ira are classD/classC fusion bifunctional beta lactamases, $\mathrm{OCH}$ is ambler class c chromosomal encoded beta lactamases, pdc are class $C$ beta lactamases, sfh is subclass B2 beta lactamase

MecRl (membrane-bound signal transduction protein) is encoded by the mecRl gene, while mecI (transcriptional regulator) is encoded by the mecI gene (MecI). The promoters for these genes are located between mecA and mecRl, as well as an operator region that includes mecA's 10 sequence and mecRl's -35 sequence. BlaRl and BlaI, which are involved in the inducible expression of the plasmid-mediated staphylococcal $\beta$-lactamase gene, blaZ, have a high protein sequence homology with MecRl and MecI, respectively. The BlaRI and BlaI genes are arranged similarly to those in the mecA system, suggesting that mecA may have acquired the regulatory genes from the blaZ system in the past. BlaI can regulate PBP2a expression since the operator regions are comparable enough. As a result, the presence of a plasmid containing the blaZ regulatory genes can cause PBP2a expression to be inducible under BlaR1 and BlaI control. blaZ gene encodes encodes $\beta$-lactamase which is synthesized when blaRl is exposed to antibiotics which leads BlaRl to cleave itself then it cleaves the repressor BlaI by acting as a protease, directly or indirectly (an additional protein, BlaR2, may be involved in this pathway)

At the Wellcome Trust Sanger Institute, genome sequencing of ALGA251 revealed that the strain harboured an unique mecA homologue, first dubbed mecALGA251, that was only 69 percent identical to conventional mecA at the DNA level and encoded PBP2a/20 was 63 percent similiar at the amino acid level.

Single-step point mutations leading in amino acid substitutions in the rpoB, (mutation) rpoC gene, and many other genetic alterations have been found to cause high-level Rimapin 
resistance. While these alterations lower the drug's affinity for its target, they usually spare the polymerase's catalytic function, allowing transcription to proceed.

The methylation of the ribosome mediated by an enzyme encoded by the erm genes (erythromycin ribosomal methylation), which leads in macrolide resistance, is an indication of resistance by enzymatic modification of the target site. ErmA is a membrane fusion protein that works with ErmB and TolC to provide an efflux pathway between E. coli's inner and outer membranes. In E. coli, ermB is a translocase in the emrB -TolC efflux protein. Carbonyl cyanide $m$ chlorophenylhydrazone (CCCP), nalidixic acid, and thioloactomycin are among the substrates it identifies. Whereas, MsrA (macrolide-streptogramin resistance), an ABC efflux protein and mef's efflux transporters confers resistance against antibiotics

\subsubsection{Specific Genes to Macrolides.}

Macrolides reduced drug binding affinity, caused by changes in either the bacterial ribosome or antibiotic molecule, and efflux of macrolides from the bacterial cell, caused by changes in me mbrane permeability or efflux pump expression, are the two most frequent resistance mechanism $s$ in bacterial .Drug inactivating mechanisms include phosphorylation of the 2'hydroxyl of the su gar by phosphotransferases and hydrolysis of the macrocyclic lactone by esterases, and ribosom emodification mechanisms include either ribosomal 23S rRNA or large ribosomal subunit protei nwhile drug inactivating mechanisms include phosphorylation of the 2 'hydroxyl of the sugar by phosphotransferases and hydrolysis of the macrocyclic lactone by esterases.

The methylation of the ribosome mediated by an enzyme encoded by the erm genes (erythromycin ribosomal methylation), which leads in macrolide resistance, is an indication of resistance by enzymatic modification of the target site. EmrA is a membrane fusion protein that works with EmrB and TolC to provide an efflux pathway between E. coli's inner and outer membranes. In E. coli, emrB is a translocase in the emrB -TolC efflux protein. Carbonyl cyanide $m$ chlorophenylhydrazone (CCCP), nalidixic acid. MsrA (macrolide-streptogramin resistance), an $A B C$ efflux protein and mef's efflux transporters confers resistance against antibiotics. MPH $\left(2^{\prime}\right)$-I is an mphA-encoded resistance enzyme that significantly inactivates macrolides. In a GTP-dependent way, it phosphorylates macrolides at the 2'-OH hydroxyl of the desosamine sugar of macrolides

\subsubsection{Specific Genes to Other Antobiotics.}

Sul is a dihydropteroate synthase gene that has been shown to be resistant to antibiotics using the antibiotic target replacement approach. Resistance to sulfonamides is mostly caused by mutations in the folP gene, which encodes the dihydropteroate synthase (DHPS) enzyme involved in nucleotide biosynthesis, or by the acquisition of alternative DHPS genes (sul1, sul2, and sul3), whose products have a low affinity for sulfonamides. However, mprF catalyses the transfer of a lysyl group from L-lysyl-tRNA(Lys) to membrane-bound phosphatidylglycerol (PG), resulting in lysylphosphatidylglycerol (LPG), a positive net charge component of the bacterial 
membrane. LPG production adds to bacterial pathogenicity since it is engaged in the host's immune system's (defensins, cathelicidins) and competing microorganisms' (bacteriocins) resistance mechanisms against cationic antimicrobial peptides (CAMP).

mphC confers resistance by phosphotransferase responsible for antiobiotic's enzyme inactivation. Whereas, dfr : dihydrofolate reductase leads to the overexpression of enzymes which will eventually reduce the affinity for antbiotics results in its resistance. On the other hand mutants of mexR nalB nalC nalD cannot repress the expression of mexAB-oprM operon confers antibiotic resistance. oprD porin protein results in non specific entry and exit points and decrease in this porin expression and altered expression of oprD and efflux pump confers resistance and mexZ inhibits mexXY multidrug transporter operon by acting as a transcriptor regulator results in resistance of antibiotic. carA exports antibiotics via energy driven ATP hydrolysis method. Tetracyclines are inactivated by the enzyme tetX, which is encoded by the tetX gene. This enzyme is unique in that it is the first and only tetracyline-inactivating enzyme ever discovered. MacA is a membrane fusion protein that, along with MacB and TolC, forms an antibiotic efflux complex. PatA is an ABC transporter that confers fluoroquinolone resistance when it interacts with PatB.

The efflux pump mexCD-oprJ is repressed by $N f x B$, which is also a repressor ( $N f x B$ binds upstream of the $n f x B$ gene and negatively regulates its own expression). Mutations in NfxB cause an increase in MexCD-OprJ expression. Overexpression of an efflux operon, mexC-mexD-oprJ, encoding components of a second efflux pump in P. aeruginosa, causes multidrug resistance in $n f x B$ strains. Whereas, modified peptidoglycan precursors ending in d-Ala-d-Lac (VanA, VanB, and VanD) or d-Ala-d-Ser (VanC, VanE, and VanG) instead D-Ala-D-Ala ligase (a former one) with low binding affinities for glycopeptides confers resistance. Resistance confers either by efflux method or by ribosomal protection. Efflux is regulated by energy-dependent efflux pumps; another crucial mechanism is a ribosome-protecting elongation factor G-like protein. Tetracycline resistance can be caused by the synthesis of a protein that interacts with the ribosome and prevents protein synthesis from being impacted by the antibiotic.

\section{CONCLUSIONS}

We hereby conclude there is a need of early detection and close monitoring of emerging resistance species of bacteria resulting into MDR bacteria. This must be started by all microbiological labs round the globe to reduce the effect of antimicrobial resistance and should be taken as problem of global interest to prevent the upcoming epidemics.

FUNDING: Department of Biotechnology (DBT), Ministry Of Science And Technology, Government Of India. 


\section{REFERENCES}

1. Bernier, Steve P., David Lebeaux, Alicia S. DeFrancesco, Amandine Valomon, Guillaume Soubigou, Jean-Yves Coppée, Jean-Marc Ghigo, and Christophe Beloin. "Starvation, Together with the SOS Response, Mediates High Biofilm-Specific Tolerance to the Fluoroquinolone Ofloxacin.” Edited by Patrick H. Viollier. PLoS Genetics 9, no. 1 (January 3, 2013): e1003144. https://doi.org/10.1371/journal.pgen.1003144.

2. Dever, Laura A. "Mechanisms of Bacterial Resistance to Antibiotics." Archives of Internal Medicine 151, no. 5 (May 1, 1991): 886. https://doi.org/10.1001/archinte.1991.00400050040010.

3. Feldgarden, Michael, Vyacheslav Brover, Daniel H. Haft, Arjun B. Prasad, Douglas J. Slotta, Igor Tolstoy, Gregory H. Tyson, et al. "Validating the AMRFinder Tool and Resistance Gene Database by Using Antimicrobial Resistance Genotype-Phenotype Correlations in a Collection of Isolates.” Antimicrobial Agents and Chemotherapy 63, no. 11 (August 19, 2019). https://doi.org/10.1128/aac.00483-19.

4. Goltermann, Lise, and Tim Tolker-Nielsen. "Importance of the Exopolysaccharide Matrix in Antimicrobial Tolerance of Pseudomonas Aeruginosa Aggregates." Antimicrobial Agents and Chemotherapy 61, no. 4 (April 1, 2017). https://doi.org/10.1128/AAC.02696-16.

5. Hall, Clayton W., and Thien-Fah Mah. "Molecular Mechanisms of Biofilm-Based Antibiotic Resistance and Tolerance in Pathogenic Bacteria.” FEMS Microbiology Reviews 41, no. 3 (March 25, 2017): 276-301. https://doi.org/10.1093/femsre/fux010.

6. Harris, Simon R, Edward JP Cartwright, M Estée Török, Matthew TG Holden, Nicholas M Brown, Amanda L Ogilvy-Stuart, Matthew J Ellington, et al. "Whole-Genome Sequencing for Analysis of an Outbreak of Meticillin-Resistant Staphylococcus Aureus: A Descriptive Study." The Lancet Infectious Diseases 13, no. 2 (February 2013): 130-36. https://doi.org/10.1016/s1473-3099(12)70268-2.

7. Holloway, Kathleen Anne, Gitanjali Batmanabane, Monika Puri, and Klara Tisocki. "Antibiotic Use in South East Asia and Policies to Promote Appropriate Use: Reports from Country Situational Analyses.” BMJ, September 5, 2017, j2291. https://doi.org/10.1136/bmj.j2291. 
8. Nguyen, D., A. Joshi-Datar, F. Lepine, E. Bauerle, O. Olakanmi, K. Beer, G. McKay, et al. "Active Starvation Responses Mediate Antibiotic Tolerance in Biofilms and NutrientLimited Bacteria." Science 334, no. 6058 (November 17, 2011): 982-86. https://doi.org/10.1126/science.1211037.

9. Pantosti, Annalisa, Fabio D’Ambrosio, Agapito Tarasi, Simona Recchia, Graziella Orefici, and Paola Mastrantonio. "Antibiotic Susceptibility and Serotype Distribution OfStreptococcus PneumoniaeCausing Meningitis in Italy, 1997-1999." Clinical Infectious Diseases 31, no. 6 (December 2000): 1373-79. https://doi.org/10.1086/317502.

10. Penterman, Jon, Dao Nguyen, Erin Anderson, Benjamin J. Staudinger, Everett P. Greenberg, Joseph S. Lam, and Pradeep K. Singh. "Rapid Evolution of Culture-Impaired Bacteria during Adaptation to Biofilm Growth.” Cell Reports 6, no. 2 (January 2014): 293-300. https://doi.org/10.1016/j.celrep.2013.12.019.

11. Pokrovskaya, Varvara, and Timor Baasov. "Dual-Acting Hybrid Antibiotics: A Promising Strategy to Combat Bacterial Resistance.” Expert Opinion on Drug Discovery 5, no. 9 (July 21, 2010): 883-902. https://doi.org/10.1517/17460441.2010.508069.

12. Randall, C. P., L. B. Oyama, J. M. Bostock, I. Chopra, and A. J. O’Neill. "The Silver Cation (Ag+): Antistaphylococcal Activity, Mode of Action and Resistance Studies." Journal of Antimicrobial Chemotherapy 68, no. 1 (September 25, 2012): 131-38. https://doi.org/10.1093/jac/dks372.

13. Schafhauser, J., F. Lepine, G. McKay, H. G. Ahlgren, M. Khakimova, and D. Nguyen. "The Stringent Response Modulates 4-Hydroxy-2-Alkylquinoline Biosynthesis and Quorum-Sensing Hierarchy in Pseudomonas Aeruginosa." Journal of Bacteriology 196, no. 9 (February 7, 2014): 1641-50. https://doi.org/10.1128/jb.01086-13.

14. Shankar, PRavi. "Book Review: Tackling Drug-Resistant Infections Globally.” Archives of Pharmacy Practice 7, no. 3 (2016): 110. https://doi.org/10.4103/2045-080x.186181.

15. Stewart, Philip S, Tianyu Zhang, Ruifang Xu, Betsey Pitts, Marshall C Walters, Frank Roe, Judith Kikhney, and Annette Moter. "Reaction-Diffusion Theory Explains Hypoxia and Heterogeneous Growth within Microbial Biofilms Associated with Chronic Infections." Npj Biofilms and Microbiomes 2, no. 1 (June 22, 2016). https://doi.org/10.1038/npjbiofilms.2016.12. 
16. Sulaiman, Jordy Evan, and Henry Lam. "Evolution of Bacterial Tolerance under Antibiotic Treatment and Its Implications on the Development of Resistance." Frontiers in Microbiology 12 (February 26, 2021). https://doi.org/10.3389/fmicb.2021.617412.

17. "The Identification, Typing, and Antimicrobial Susceptibility of Pseudomonas Aeruginosa Isolated from Mink with Hemorrhagic Pneumonia." Veterinary Microbiology 170, no. 3-4 (June 4, 2014): 456-61. https://doi.org/10.1016/j.vetmic.2014.02.025.

18. Walters, M. C., F. Roe, A. Bugnicourt, M. J. Franklin, and P. S. Stewart. "Contributions of Antibiotic Penetration, Oxygen Limitation, and Low Metabolic Activity to Tolerance of Pseudomonas Aeruginosa Biofilms to Ciprofloxacin and Tobramycin." Antimicrobial Agents and Chemotherapy 47, no. 1 (January 1, 2003): 317-23. https://doi.org/10.1128/aac.47.1.317-323.2003.

19. Westblade, Lars F., Jeff Errington, and Tobias Dörr. "Antibiotic Tolerance.” Edited by John M. Leong. PLOS Pathogens 16, no. 10 (October 15, 2020): e1008892. https://doi.org/10.1371/journal.ppat.1008892.

20. Zhang, Lianbo, Wen-Chi Chiang, Qingguo Gao, Michael Givskov, Tim Tolker-Nielsen, Liang Yang, and Guang Zhang. "The Catabolite Repression Control Protein Crc Plays a Role in the Development of Antimicrobial-Tolerant Subpopulations in Pseudomonas Aeruginosa Biofilms." Microbiology 158, no. 12 (December 1, 2012): 3014-19. https://doi.org/10.1099/mic.0.061192-0. 